\title{
Nanotechnology Advances in Medicine: Focus on Cancer
}

\author{
Yuna Qian, ${ }^{1,2}$ Shengzhe Zhao, ${ }^{2}$ Haifa Shen ${ }^{3,4}$ and Jianliang Shen ${ }^{1,2^{*}}$
}

In the past decades, with the notable and rapid development of nanotechnology, nanomaterials have been applied in various research fields and industries, including in medicine. The unique chemical, physical and biological properties of nanomaterials successfully address the barriers of drug delivery, improve the therapeutics, and enhance the accumulation of the functional nanoparticles in targeted tissues with amplified signals for probes or bio-images. This review summarized the updated progresses of nanomaterials serve as drug delivery and theranostic platforms for cancer therapeutics and diagnosis.

Keywords: Nanotechnology; Nanomedicine; Drug delivery; Therapeutics; Diagnosis

Received 2 January 2019, Accepted 28 January 2019

DOI: $10.30919 / \mathrm{es} 8 \mathrm{~d} 678$

\section{Introduction}

Nanomedical engineering has grown remarkably in the past decades with the concurrent rise of biomedical engineering and nanotechnology. It involves the manipulation of matter in the size range of 1-1000 nm for medical applications, which generally can be categorized as therapeutic agents (carriers for drug delivery) or medical imaging agents. ${ }^{1}$ Compared to existing drugs and agents, this new kind of nanotechnology is attractive due to their unique characteristics, including large surface area, structural properties, and long circulation time in blood. ${ }^{2}$ Potential advantages of engineered nanomedical agents are the ability to: improve drug delivery across biological barriers and compartments; control the release of bioactive agents; enhance the therapeutic effect by targeted delivery; and perform theranostic functions by combining multimodal imaging and simultaneous diagnosis and therapy into multifunctional nanoplatforms. ${ }^{3-5}$

A large research focus of nanomedicines has been placed on cancer treatments. Nowadays, cancer is still a main leading cause of death worldwide, largely due to the heterogeneous and idiosyncratic nature of individual cancers, and the inability to target therapeutics to neoplastic areas without damaging normal tissues. ${ }^{6}$ Among the emerging technologies, nanotechnology provides a plethora of promising avenues for cancer theranostics, especially for drug delivery to solid tumor. Nanomedicine compounds can alter the biodistribution

${ }^{1}$ School of Ophthalmology \& Optometry, School of Biomedical Engineering, Wenzhou Medical University, Wenzhou, Zhejiang 325035, China

${ }^{2}$ Wenzhou Institute of Biomaterials and Engineering, Chinese Academy of Science, Wenzhou, Zhejiang 325001, China

${ }^{3}$ Department of Nanomedicine, Houston Methodist Research Institute, Houston, Texas 77030, USA

${ }^{4}$ Department of Cell and Developmental Biology, Weill Cornell Medicine, New York, NY10065, USA

*E-mail: shenjl@wibe.ac.cn of drugs by allowing them to accumulate preferably at the tumor site attributed to leaky tumor vasculature and impaired lymphatic drainage. This phenomenon is known as enhanced permeability and retention effect (EPR).

A wide range of nanomaterials based on various components from metals to proteins, including carbon, silica oxides, metal oxides, nanocrystals, lipids, polymers, dendrimers, and quantum dots, as well as newly developed materials, have been employed for the development of new cancer therapeutics. ${ }^{8-12}$ There are several excellent review articles on the biomedical applications of these nanomaterials. Although these materials are likely to provide a high degree of biocompatibility, several issues should be considered to translate developed nanomedicines into clinical practice, such as off-target effects, organism effective clearance or toxicity to healthy tissues. During the past several years, our group has devoted enormous efforts to develop various inorganic and organic nanomaterials, including $\beta$-cyclodextrin $(\beta-C D)$, porous silicon, quantum dots, and gold nanoparticles, etc. And we designed a variety of nanoplatforms for siRNA delivery, chemo-therapy, codelivery of multiple therapeutics, probe and bio-imaging in cancer therapy.

Therefore, in this review, we shall discuss our own work made in the designed fabrication of nanoparticles for the multifunctional platform as drug carriers and tumor diagnosis. Nanoparticles design and surface modification targeting the dynamic interplay of the tumor microenvironment will be highlighted, which aiming to summarize some of the past works, and to analyze the challenges and opportunities at present.

\section{Nanotechnology Advances in therapeutic agents}

\section{1. siRNA delivery by nanomaterials}

As the new anti-cancer therapeutics, small interfering RNA (siRNA) and RNA interference (RNAi) have been widely recognized to be a promising biotechnology. ${ }^{13}$ Despite the great versatility of RNA interference (RNAi) technology, which can down-regulate any protein in targeted cells and tissues, many physiological and biological obstacles still stand in the clinical application, such as negative charges, short half-life and low stability. ${ }^{14}$ These issues can be overcome by 
chemical modifications of the RNA backbone or by embedding the siRNA into nano-carriers. Up to now, a great number of systems were reported for delivery siRNA including a new, non-viral way to overcome bio-barriers. ${ }^{15}$ Here we summarize the siRNA delivery systems by metallic and non-metallic core hybrids with organic moieties.

Non-viral siRNA delivery hybrid nanoparticles have shown promise and have been used in clinical trial. These functional systems have the advantages, such as low/non-toxicity, easy fabrication, biodegradation, biocompatibility, non-immunogenicity, low price and high efficacy comparing with the commercial agents. ${ }^{16}$ Gold nanoparticle (NP) is the typically metal-based NP for the siRNA delivery. ${ }^{17}$ Gold is known to be one of the least reactive metals, exhibiting incredible chemical resistance against both oxidation and corrosion. The biocompatibility of $\mathrm{Au}$ nanomaterials has been widely demonstrated by a number of in vitro and in vivo studies. However, the modification of the surface of gold NPs is essential before they can be used for biomedical applications. We have used Au NRs as a delivery platform for siRNA gene silencing agents. The surface of Au NRs was modified with polyethylenimine (PEI) through layer-by-layer assembly and siRNA was absorbed on the surface (Fig. 1). ${ }^{18}$ We demonstrated that PEI-Au NRs not only protect siRNA from degradation, but also facilitate endosomal escape, both of which are prerequisites for successful gene silencing.

The other serial of the hybrid Nano systems is non-metal based core with further functional organic shells such as silica/silicon. ${ }^{19}$ Precise nanopore formation and easily surface modification are the main factors that brought interest to silicon and silica nanoparticles. Silica nanoparticles are biocompatible, biodegradable and efficiently bioeliminated in vivo. In order to develop a tumor-specific delivery system, a nanoparticle-in-microparticle multistage vector (MSV) delivery system with the sustained release of siRNA oligos have been developed (Fig. 2). ${ }^{20}$ This kind of MSV composed of two carriers: the first-stage carriers are mesoporous microscale biodegradable silicon particles, allowing for the loading and release of second-stage nanocarriers in a sustained manner. The second stage includes micelles, liposomes, polymeric nanoparticles or metallic nanoparticles, etc. The third stage is the payload drugs including siRNA, chemotherapeutics, miRNA, and so on.

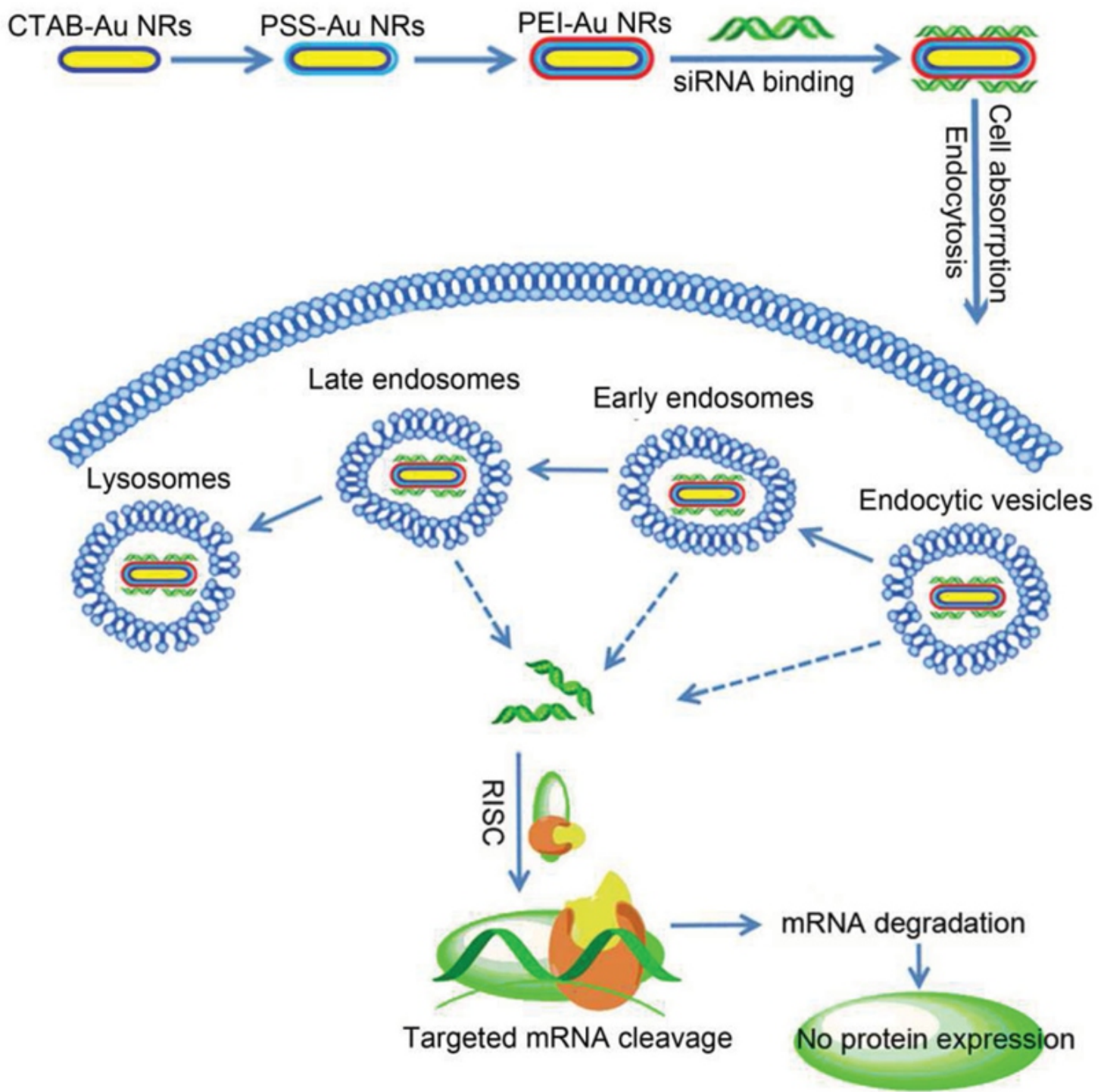

Fig. 1 Schematic illustration of the fabrication and function of PEI-Au NRs for the delivery of siRNA. (Reprinted with permission from Ref. [18]. Copyright 2014, Wiley Online Library.) 


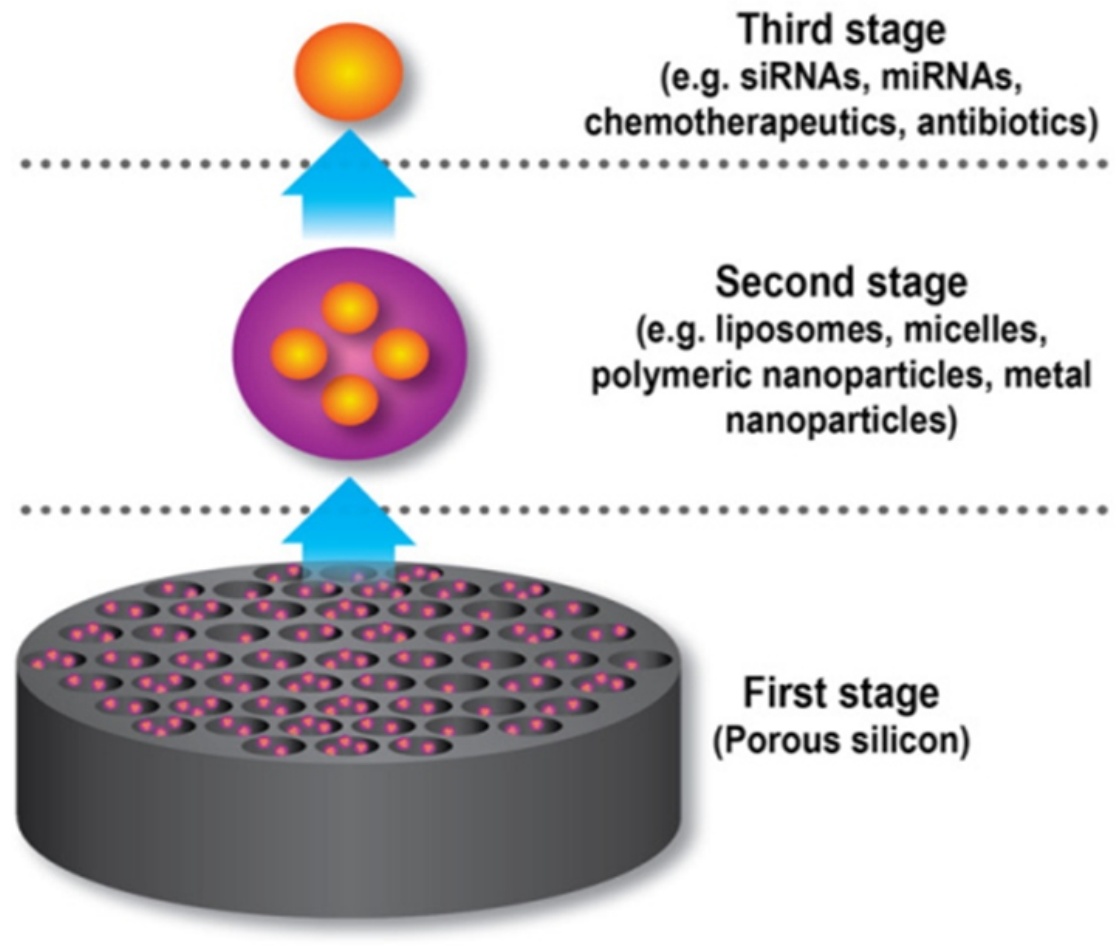

Fig. 2 The MSV consists of three components: a porous silicon microparticle, nanoparticles, and therapeutic agents. (Reprinted with permission from Ref. [20]. Copyright 2010, Elsevier.)
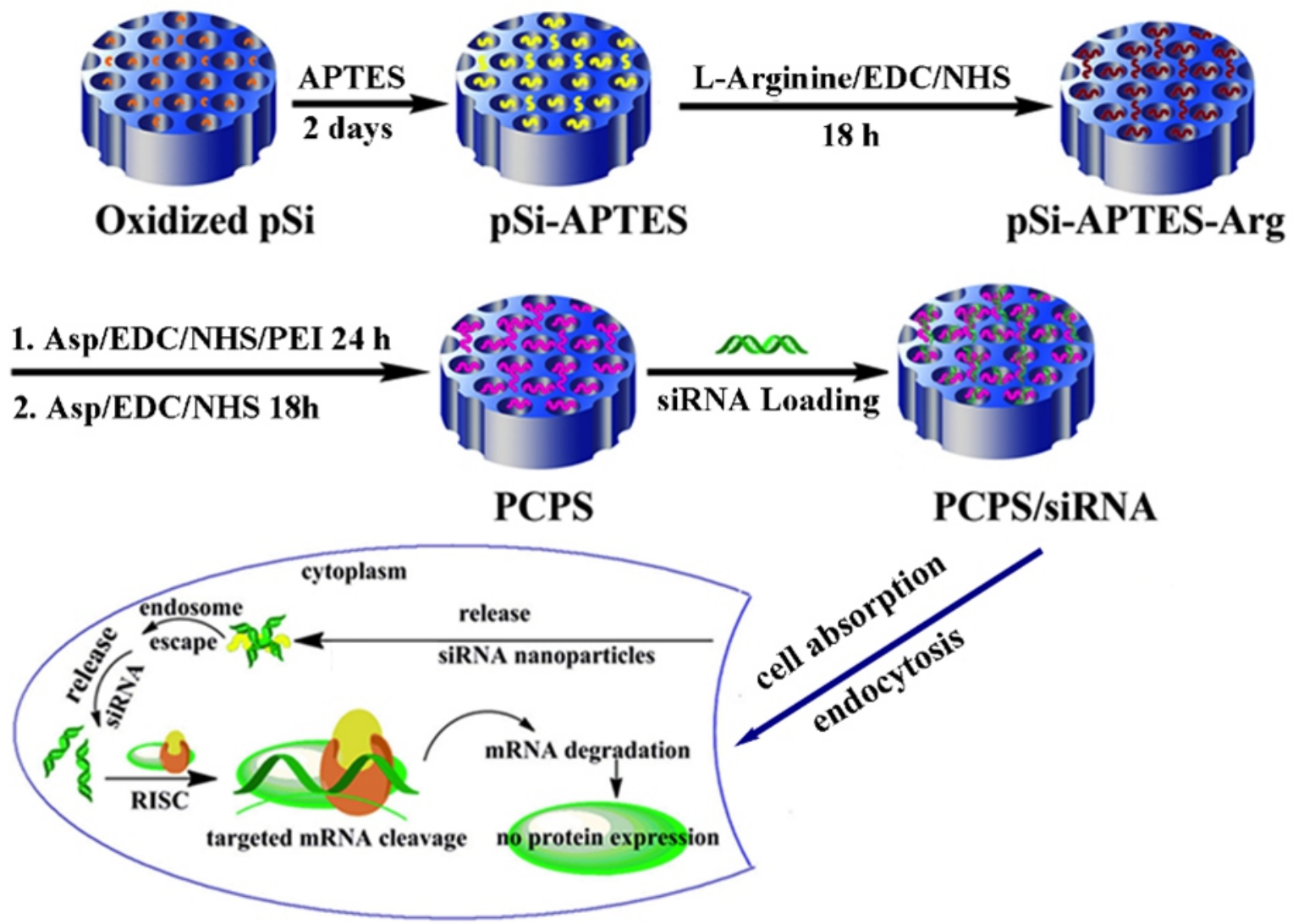

Fig. 3 Polycation nanoporous silicon (PCPS) releases self-assembled secondary nanoparticles for siRNA delivery. (Reprinted with permission from Ref. [21]. Copyright 2013, American Chemical Society. ) 


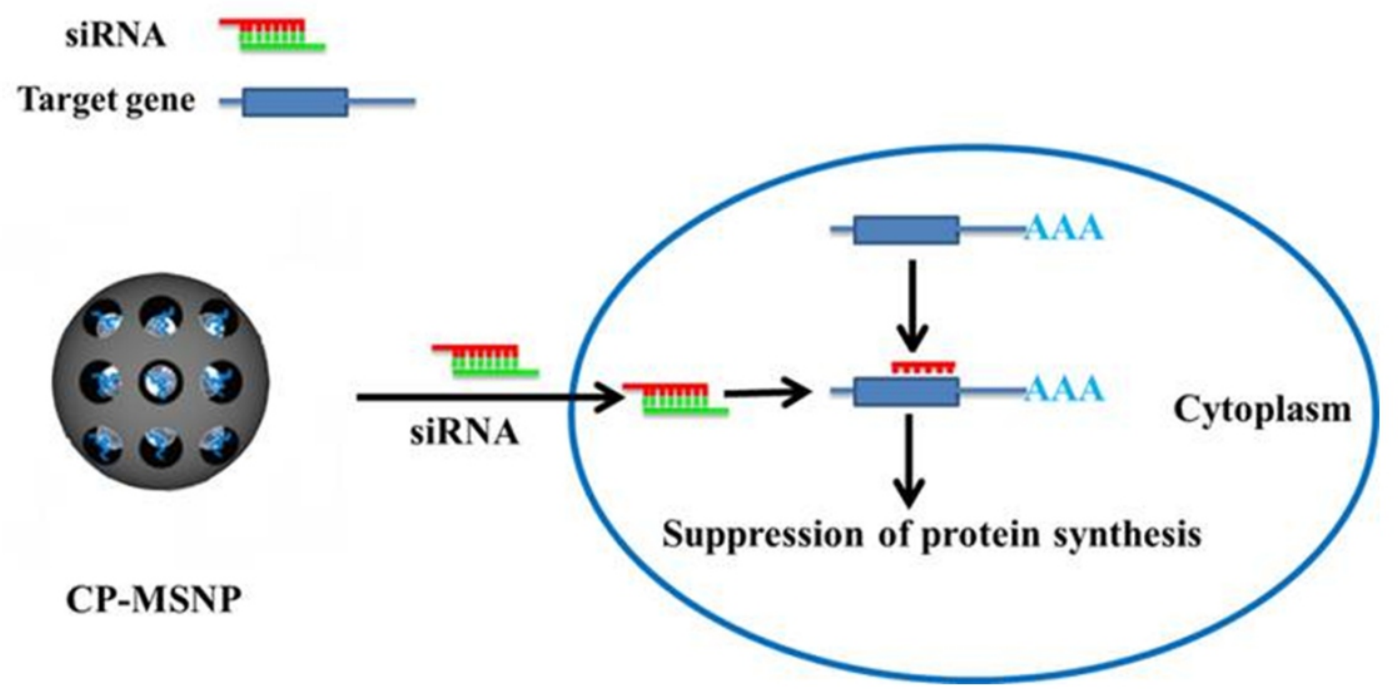

Fig. 4 Schematic illustration on fabrication of the CP-MSNP delivery carrier for gene silencing agents. (Reprinted with permission from Ref. [22]. Copyright 2014, Ivyspring.)

Based on MSV systems, we further develop a high capacity polycation-functionalized nanoporous silicon (PCPS) platform consisting of new-style second stage complexes which can form nanoparticles over time as the first-stage carrier degrades (Fig. 3). ${ }^{21}$ This platform has the following advantages: (1) high loading capacity, (2) low or no toxicity, (3) a friendly production protocol, and (4) stability during transportation and storage. Additionally, the self-assembled nanoparticles could successfully help siRNA polyplexes to escape from the endosome and lysosome to the cytoplasm with the benefits from the PEI moieties triggering the proton sponge effects. Furthermore, systemic delivery of PCPS/STAT3 siRNA in the murine model of MDAMB-231 breast cancer enriched particles in tumor tissues and reduced STAT3 expression in cancer cells, causing significant reduction of cancer stem cells in the residual tumor tissue. Totally, these inorganic (silicon) and organic (liposome or polymer) hybrid systems offers a safe vehicle for effective delivery of gene silencing agents. We also develop a universal siRNA carrier consisting of a mesoporous silica nanoparticle (MSNP) functionalized with cyclodextrin-grafted polyethylenimine (CP) (Fig. 4). ${ }^{22} \mathrm{CP}$ provides positive charge for loading of siRNA through electrostatic interaction and enables the effective endosomal escape of siRNA. Using intravital microscopy we were able to monitor tumor enrichment of CP-MSNP/siRNA particles in live mice bearing orthotopic MDA-MB-231 xenograft tumors. CPMSNP delivery of siRNA targeting the M2 isoform of the glycolytic enzyme pyruvate kinase (PKM2) resulted in effective knockdown of gene expression in vitro and in vivo. Suppression of PKM2 led to inhibition of tumor cell growth, invasion, and migration.

\subsection{Chemo-therapy by nanomaterials}

Cancer treatments have been significantly refined due to an increased awareness of the molecular, cellular, and physiological mechanisms involved in the initiation and progression of the disease. ${ }^{23}$ Small molecule drugs are still one of the most important modalities of cancer treatment, however it is often accompanied by systemic side effects including poor solubility, stability and pharmacokinetics of drugs, distribution to nontumor tissues, hemorheological flow limitations and multidrug resistance (MDR). ${ }^{24}$ The use of nanocarriers usually improves therapeutic efficacy and reduces side effects, as it can reduce renal clearance, prolong blood circulation, and improve tumor accumulation. ${ }^{25}$ In this section, several representative drug delivery systems for chemo-therapy are summarized.

Among some of the most widely studied delivery platforms, mesoporous silica nanoparticles (MSNPs) have attracted attention owing to their large pore volume and surface area, good biocompatibility, easily functionalized surface, and high cellular uptake efficiency. ${ }^{26}$ Many studies have focused on the modification of MSNPs for effective drug delivery and gene transfection. We also have reported an injectable nanoparticle generator (iNPG) that overcomes multiple biological barriers to chemo-therapy delivery. ${ }^{27}$ The $\mathrm{iNPG}$ is a discoidal micrometer-sized MSNPs that can be loaded with chemotherapeutics. We conjugate doxorubicin to poly(1-glutamic acid) by means of a $\mathrm{pH}$ sensitive cleavable linker, and load the polymeric drug (pDox) into iNPG to assemble iNPG-pDox (Fig. 5). Once released from iNPG, pDox spontaneously forms nanometer-sized particles in aqueous solution. Intravenously injected iNPG-pDox accumulates at tumors due to natural tropism and enhanced vascular dynamics and releases pDox nanoparticles that are internalized by tumor cells. Intracellularly, pDox nanoparticles are transported to the perinuclear region and cleaved into Dox, thereby yielding high intracellular concentrations of activated Dox in a cellular domain beyond the reach of cell-surface drug efflux pumps.

Up to now, carbon-based nanomaterials (CBNs) have also been actively investigated due to their advantageous chemical and physical properties (i.e., thermal effect, electrical conductivity, and high mechanical strength). ${ }^{16}$ This perspective highlights different types of carbon-based nanomaterials such as carbon nanotubes (CNTs), graphene, fullerene, carbon quantum dots and carbon fibers currently used in biomedical applications. ${ }^{28}$ We use carbon nanotubes (CNTs) as the model, through an analogous layer-by-layer assembly method, on which uniform mesoporous silica shells can successfully be formed. ${ }^{29}$ Carbon nanotubes allow to easily cross the plasma membrane and translocate into the cytoplasm of cells due to their particular needle shape. Therefore, these nanotube-based drug transfer vectors have shown minimal cytotoxicity and effective delivery.

\subsection{Codelivery of multiple therapeutics by nanomaterials}

Cancer progression involves dynamic changes in the genome and a complex network of interactions among cancer cells with multiple, distinct cell types that form tumors. The complexity of the signaling 

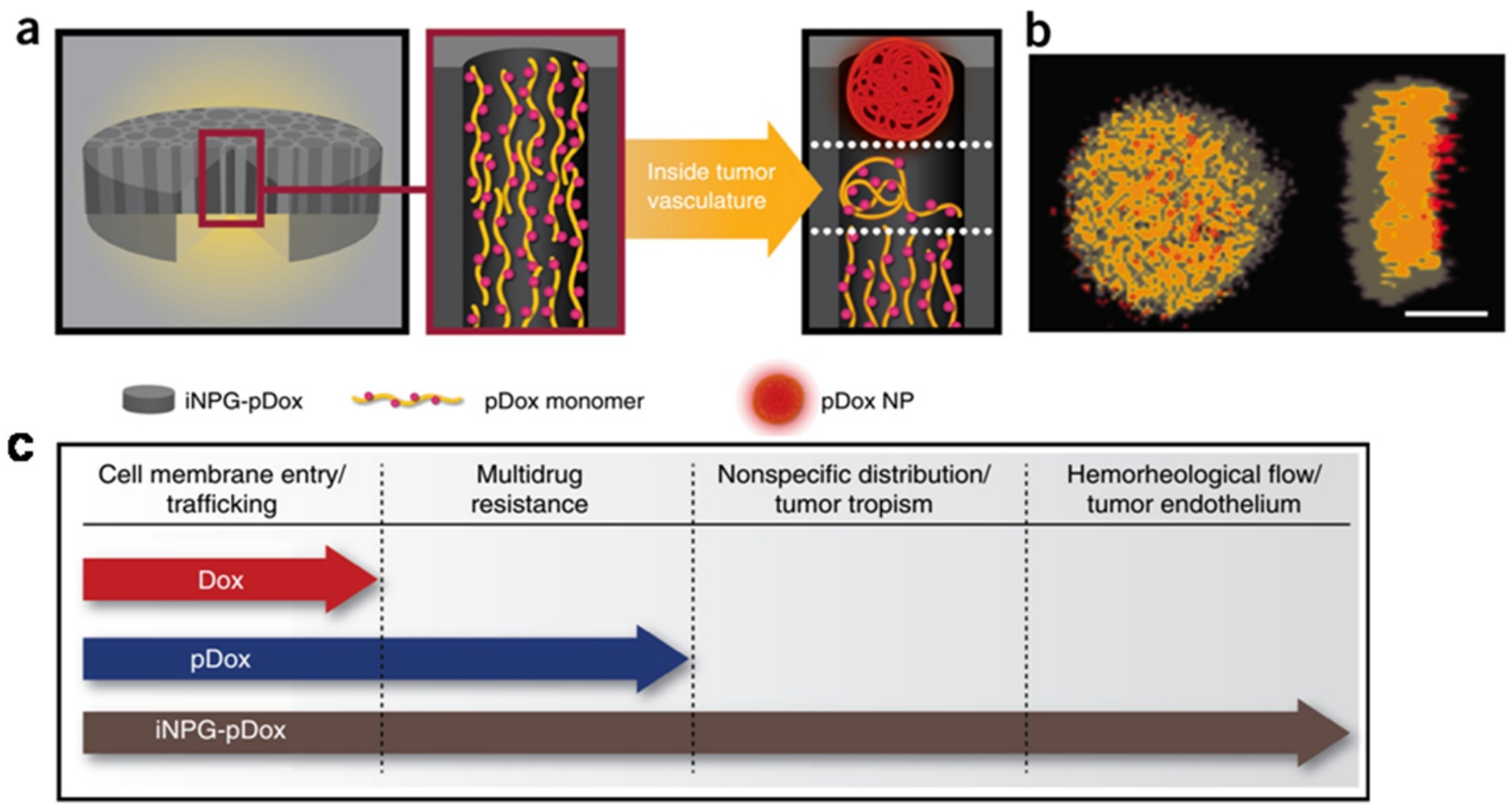

Fig. 5 iNPG-pDox characterization and pDox assembly and release from iNPG. (a) Schematic diagram depicting iNPG-pDox composition, pDox prodrug encapsulation, and pDox nanoparticle assembly and release from nanopores. (b) Z-series confocal microscopy imaging of the iNPG-pDox particles, highlighting the presence of pDox (red) within the nanopores of the silicon carrier particle (gray). Scale bar, $1 \mu \mathrm{m}$. (c) The schematic diagram demonstrating the individual components of the iNPG-pDox construct and the distinct biological barriers that each component is capable of overcoming following systemic administration. (Reprinted with permission from Ref. [27]. Copyright 2015, Nature Publishing Group.)

web, combined with multiple mechanisms to evade programmed cell death, makes curing cancer a formidable challenge. As a result, cancer treatment that uses a combination of approaches with the ability to take advantage of distinct mechanisms of action of the multiple therapeutics/modalities has been proven highly effective in the treatment of many cancers. ${ }^{30}$ However, little attention has been paid to the potential of simultaneous delivery of combinations of traditional small molecule drugs with nucleic acids clinically. This lack of attention is obvious in the limited selection of suitable vectors due to differences in the physicochemical properties of the two types of agents. Here, we discuss our group recent advances in the development of nanomaterials using combinations of small molecule drugs and nucleic acid therapeutics to treat cancer.

In order to overcome MDR in cancer cells, we have designed a multifunctional porous silica-based nanoplatform for the codelivery of chemotherapeutic agents and siRNA to tumor tissue. This platform consists of cyclodextrin-grafted polyethylenimine (CP) functionalized mesoporous silica nanoparticles (MSNP) (Fig. 6). ${ }^{31}$ A unique multi-step encapsulation procedure was used to obtain a high loading capacity for doxorubicin (DOX) and siRNA oligos. The first step was loading of DOX inside the pores of the MSNP, and the second step was capturing of DOX in the hydrophobic cavities of $\mathrm{CD}$, which was conjugated to the surface of MSNPs. Additionally, MSNPs were functionalized with CD-grafted PEI to facilitate PKM2 siRNA loading through electrostatic interactions. The in vivo and in vitro results from this study demonstrate that gene silencing and cancer cell apoptosis was achieved. In particular, suppression of PKM2 expression was shown to sensitize breast cancer cells to DOX. Taken together, this study lays the foundation for further development of porous silica-based nanoparticles for codelivery of chemotherapy and gene silencing agents.

Although particles in the nano-size range display favorable transport properties compared to small molecules, the bulk of the injected material lacks therapeutic activity. This excess material could by itself pose a safety concern. Additionally, it is probable that cells will eventually reach a limit in regards to the uptake capacity of drug carriers. In cases where this internalization limit is reached before the therapeutic threshold of the drug is obtained, the loading capacity will be a major limitation for therapeutic efficacy. These challenges have motivated the development of self-delivery systems that are formed by self-assembly of different therapeutic agents, including chemotherapy, proteins, and small interfering RNA (siRNA). ${ }^{32}$ This strategy eliminates the need for carriers that lack therapeutic properties and provides an effective means for developing synergistic combination therapies, enhancing loading capacity, and exploiting nanoparticle transport properties (Fig. 7). These self-delivery systems also highlight that the distinction between carrier and drug should become obsolete from a regulatory perspective. Recent research has demonstrated that siRNA could self-assemble with polymeric metformin (PolyMet), forming around $80 \mathrm{~nm}$ particles. ${ }^{33}$ Metformin has shown promise as an anticancer agent in multiple clinical trials, which could increase cellular uptake, enhance transfection, and circumvent efflux pumps. The results indicated that nanoparticle-treatment caused a dramatic reduction in tumor growth compared to control groups. Specifically, polymeric metformin serves both as a therapeutic agent and carrier for siRNA. This strategy paves the way for the next generation of therapeutic nanoparticles for the treatment of disease. 


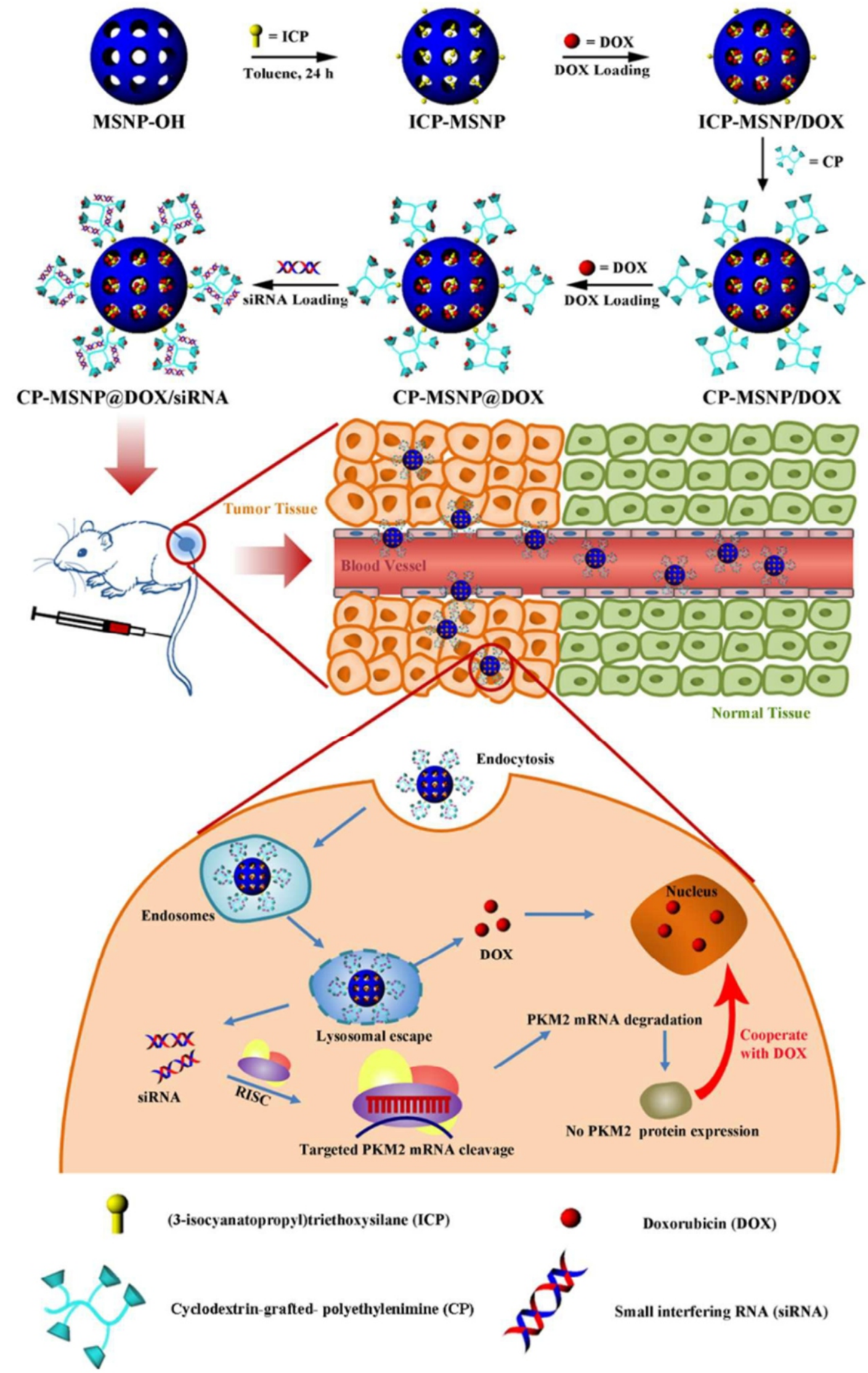

Fig. 6 Schematic representations of the fabrication of CD-grafted PEI-mesoporous silica nanoparticles@Dox/siRNA as a codelivery system for triplenegative breast cancer (TNBC). ICP, (3-isocyanatopropyl)triethoxysilane. (Reprinted with permission from Ref. [31]. Copyright 2017, Royal Society of Chemistry.) 


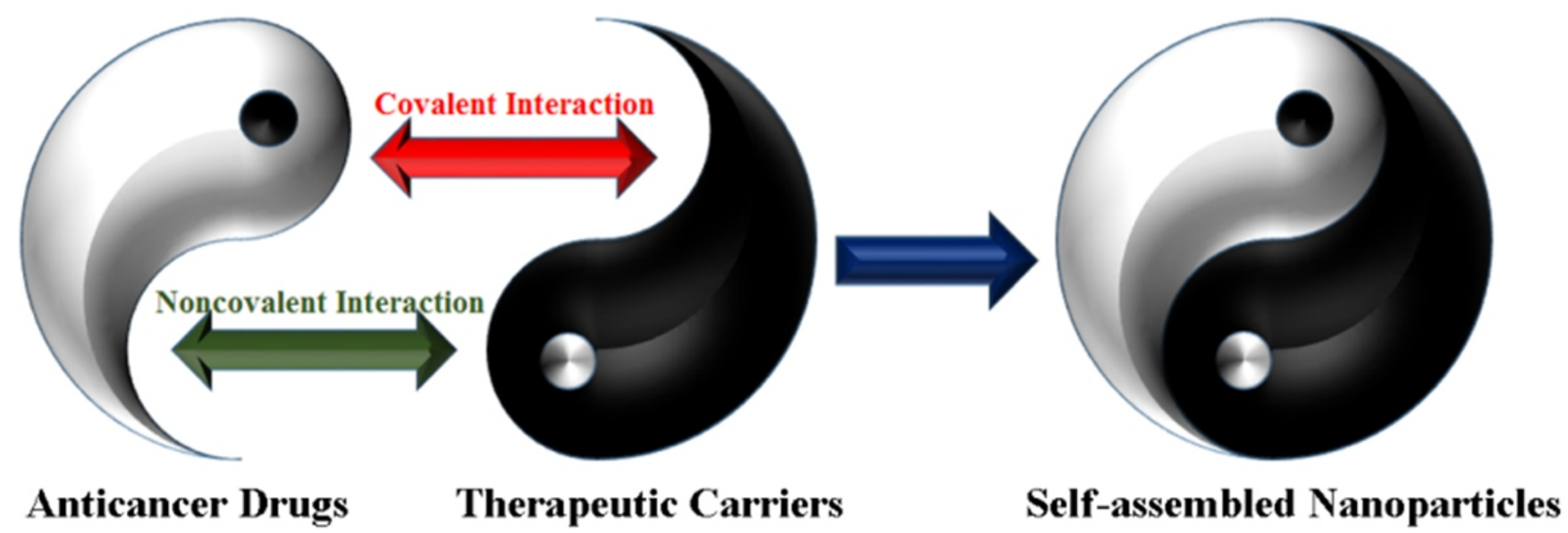

Fig. 7 Schematic representation of Self-delivery systems for combination therapy. (Reprinted with permission from Ref. [32]. Copyright 2017, Elsevier.)

\section{Nanotechnology advances in disease detection and diagnosis}

\subsection{Probe or bio-imaging}

Many cancers would be curable or at least treatable if it can be diagnosed at its earliest stage. In this regard, molecular imaging with targeted contrast agents, such as nuclear and optical probes, can play a key role in cancer screening, detection, and management. ${ }^{34}$ In addition to meeting the broad interest in the area of drug delivery, nanotechnology is also playing a leading role in biomedical imaging especially in medical conditions such as cancer. The combination of nanotechnology and molecular imaging provides a versatile platform for novel (nano-) probe design, which can enhance the sensitivity, specificity and signal amplification of traditional imaging agents. ${ }^{35}$

luminescent quantum dots (QDs) have become well-established fluorophores in various biomedical areas since the pioneering works in 1998. ${ }^{36}$ QDs exhibit unique optical properties, such as size-tunable emission, resistance to photobleaching, superior signal brightness, and broad absorption spectra of excitation. Therefore, QDs have been considered to be promising nanoscale scaffolds for designing multifunctional nanosystems with a nanomedical probe or bio-imaging functions. For instance, it is possible to label proteins with selective fluorescent, which is traditionally classical immunocytochemistry done. In addition, quantum dots present minimal photobleaching and a much higher signal to noise ratio compared to traditional methods. They have a wide absorption spectrum while maintaining very narrow emission spectra, allowing multiplexing of many quantum dots of different colors in the same sample. ${ }^{37}$ This is unique and cannot be achieved using traditional fluorophores.

When used QDs as a probe for detecting biological samples, the water-solubility and biocompatibility are very essential. ${ }^{38}$ The most common strategy to make QDs water-soluble is anchoring thiol (-SH) functionalities that are reactive to the surface of QDs. However, it was found that they do not efficiently stabilize QDs, and can result in significant drop in quantum yields (QYs) in biological buffers or culture media. Furthermore, they were highly toxic to living cells. ${ }^{39}$ Our group prepared a series of QDs (CdSe, CdSe/CdS and $\mathrm{CdSe} / \mathrm{ZnSe}$ ), coated with L-arginine-functional beta-cyclodextrin (Fig. 8). ${ }^{40,41}$ We observed that b-CD-L-Arg-coated QDs are water-soluble and stable with high colloidal properties in water. Their photophysical properties are similar to those of trioctylphosphine oxide (TOPO)-coated nanocrystals. Most important of all, the in vitro cytotoxicity analysis in ECV-304, SHSY5Y and Hela cells showed these QDs presented lower toxicity to living cells. A series of different kinds of amino acid $\left(_{L}-H i s,{ }_{L}-T r p,{ }_{L}-P h e\right.$, ${ }_{L}$-Cys)-modified $\beta$-CD-coated QDs also were prepared to study the luminescence and biocompatibility properties. ${ }^{42}$ It was found that ${ }_{\mathrm{L}}-\mathrm{His}-$ $\beta$-CD-coated QDs had the best optical properties and colloidal stability. In particular, ${ }_{\mathrm{L}}$-His- $\beta$-CD-coated QDs were the least cytotoxic. These results show that the QDs coated by amino acid-modified $\beta-C D$ can be used as a potential photoluminescent nanocrystal probe with good biocompatibility.

\subsection{Theranostic platform}

Clever combinations of different kinds of functional nanostructured materials will enable the development of multifunctional nanomedical platforms for multimodal imaging or simultaneous diagnosis and therapy, referred as theranostics. ${ }^{43}$ Theranostic agents based on nanoparticles will enable monitoring of drug delivery carrier, such as measuring delivery efficiency, selectivity, sites of localization of drugs/genes, and concurrent measurement of the drug treatment response (such as reduction in tumor volume). ${ }^{44}$ In addition, in situ imaging of drug delivery nanoparticles can provide startling insights about the molecular mechanism of tumor progression (such as its invasion and metastatic behavior), and can help in the future design and development of novel molecular nanoprobes-based delivery systems. ${ }^{34}$ These advanced multifunctional theranostics nanosystems are thus envisaged to play a major role in the area of personalized cancer therapy.

Theranostic nanomedicine can work better than other theranostics since they have advanced capabilities in an all-in-one single platform, which include controlled release, targeted delivery, synergetic performance and multimodality diagnosis. ${ }^{43}$ Interestingly, siRNA can also be included in theranostic nanomedicine as an inhibitor of theranostic resistance. ${ }^{45}$ The siRNA based theranostic nanomedicine has shown to have greatly improved the diagnosis and therapy as multimodality therapy. With excellent fluorescence properties, siRNA nanocarriers engineered from QDs offer great advantages for targeted siRNA delivery tracking, mechanistic studies and therapeutic efficiency evaluation. Mao's group has reported a series of biocompatible QDs modified with amino acid grafted $\beta$-cyclodextrin $(\beta-C D)$ derivatives. The arginine- $b-C D$ modified QDs (Arg-CD-QDs) not only have a 


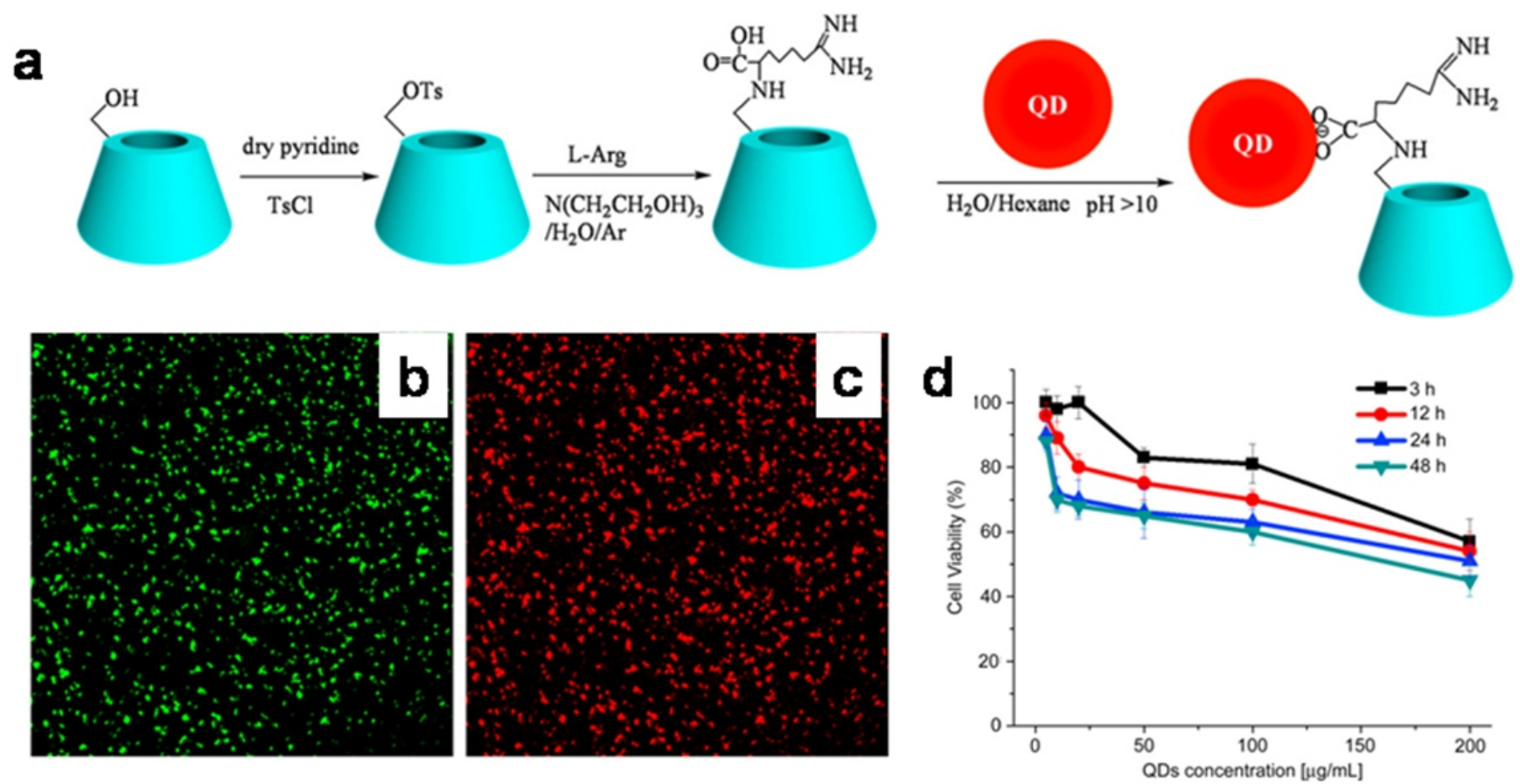

Fig. 8 (a) Schematic illustration of the formation of the b-CD-L-Arg-coated QDs nanocrystals, (b) Luminescent images of b-CD-L-Arg/CdSe/ZnSe QDs cultured with ECV-304 cells with green emission, (c) with red emission, (d) Effects of b-CD-L-Arg/CdSe/ZnSe QDs on ECV-304 cells at different time points. (Reprinted with permission from Ref. [40]. Copyright 2010, Elsevier.)
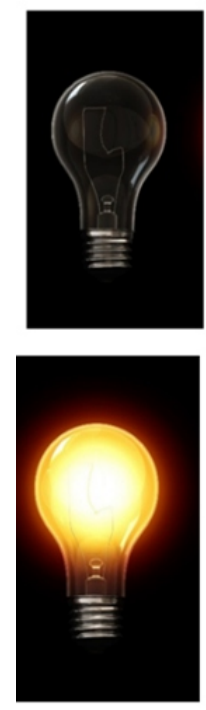

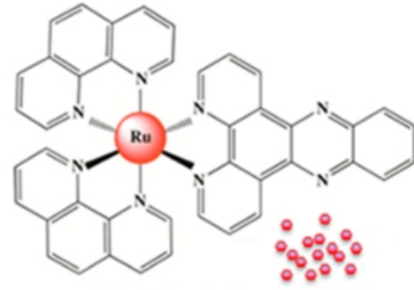

$\left[\mathrm{Ru}(\text { phen })_{2} \mathrm{dppz}\right]\left(\mathrm{ClO}_{4}\right)_{2}$

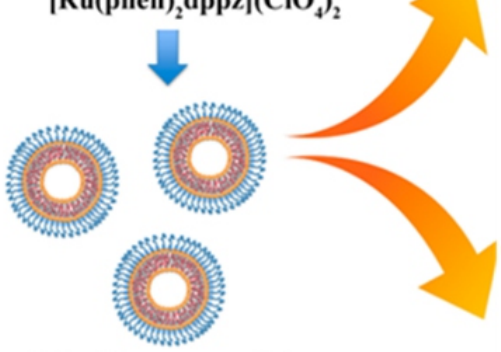

Lipo-Ru nanoparticles

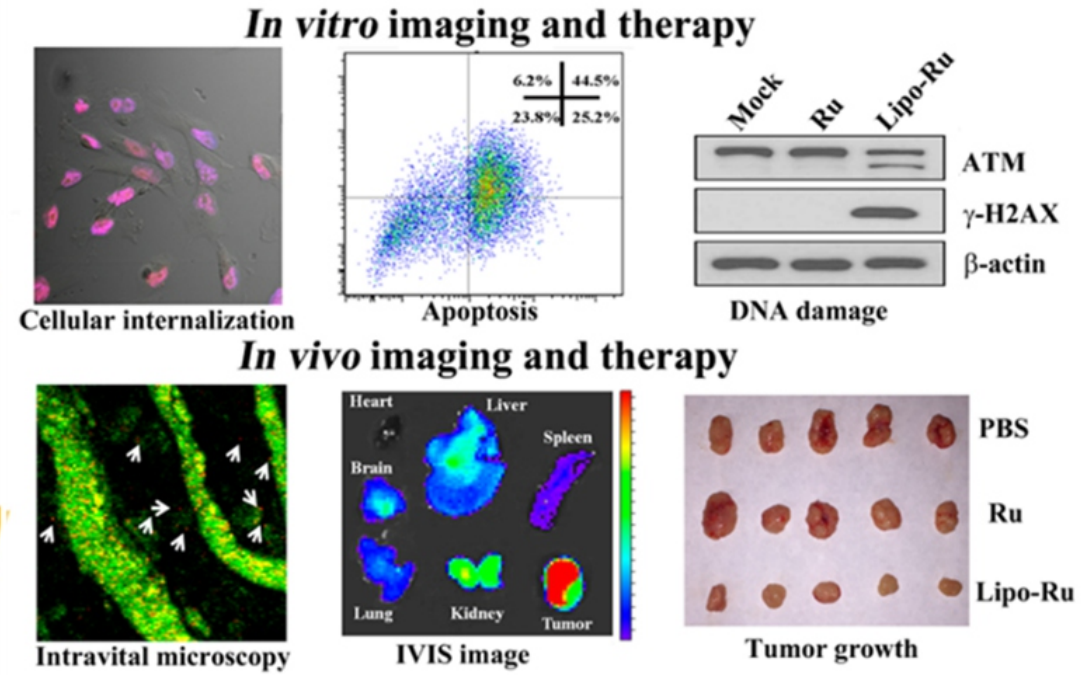

Fig. 9 Schematic Representation of Lipo-Ru with the in vitro and in vivo imaging and therapy. (Reprinted with permission from Ref. [47]. Copyright 2017, American Chemical Society.)

positively charged surface to absorb siRNA, but also a hydrophobic cavity of $b-C D$, which serves as a molecular capsule to encapsulate doxorubicin (Dox). ${ }^{46}$ The siRNA was designed to target and silence the multidrug resistance gene (MDR-1), which is responsible for multidrug resistance in cancer cells. By simultaneous transportation of siRNA, DOX and QDs across the cell membrane to down-regulate the expression of P-glycoprotein (P-gp), the multidrug resistance could be reversed and as a result the efficiency of theranostics complexes can be improved. At the same time, these QDs complexes could also be used as nanocrystal probing agents, allowing real-time tracking and localization of drugs during delivery and transfection.

Many of the biocompatible polymers are suited to encapsulate multiple types of imaging probes and drugs in high concentrations/loading. We have developed a liposome-based theranostic nanodelivery system for $\left[\mathrm{Ru}(\mathrm{phen})_{2} \mathrm{dppz}\right]\left(\mathrm{ClO}_{4}\right)_{2}(\mathrm{Lipo}-\mathrm{Ru})$ (Fig. 9). ${ }^{47} \mathrm{Ru}$ has a range of unique oxidation states (II, III, and IV) that contribute to the anticancer activity. Besides exhibiting anticancer activity, Ru complexes also display the potential to be used as imaging agents by binding to DNA through noncovalent interactions. The lipid bilayer of our system provides a hydrophobic environment that causes 
$\mathrm{Ru}$ to continuously emit fluorescence light, thereby enabling nanoparticle tracking inside the body. Notably, once the drug is released from the liposomes, it continues to generate florescence by binding to DNA. Incubation of MDA-MB-231 breast cancer cells with Lipo-Ru induced double-strand DNA breaks and triggers apoptosis. In a mouse model of triple-negative breast cancer, treatment with Lipo-Ru dramatically reduced tumor growth. In conclusion, this study has confirmed the potential of Lipo-Ru to serve as an efficient theranostic platform and the results suggest that this system could have broad applicability across a variety of solid tumors.

\section{Conclusion and Perspectives}

The unique properties of carefully designed nanomedicines hold great potential for the treatment of cancer. In this chapter we present some of our own technologies in nanoplatform that have the potential to drug delivery and imaging including gold nanoparticles, porous silicon, carbon nanotubes, QDs and $\mathrm{Ru}$ complexes. These nanoplatforms provide opportunities to overcome challenges such as poor solubility, stability, biocompatibility, distribution to nontumor tissues, hemorheological flow limitations and multidrug resistance (MDR) on cancer therapy. However, several challenges still need to be overcome in order for these nanostructured materials to achieve translation into the clinical setting. In particular, more thorough preclinical assessment of nanomedicines must be conducted. Until recently, methods for studying nanoparticle intratumoral behavior were limited. ${ }^{25}$ Besides, the in vivo clearance and potential long-term immune responses of nanoparticles should be more exploited. Further, recent researches have demonstrated tumor stromal cells (cancer-associate fibroblasts, macrophages, etc) play a crucial role in nanomedicine delivery and significantly alter particle pharmacokinetics and bioavailability. If these issues are satisfactorily addressed in the future, these multifunctional nanoplatforms will provide important new tools for simultaneous diagnosis and efficient and specific treatment of diseases in the clinical practices.

\section{Acknowledgements}

This work was financially supported by the National Natural Science Foundation of China (31800833), Zhejiang Provincial Natural Science of Foundation of China (Z19H180001 and LQ19C100001), the Wenzhou Medical University and Wenzhou Institute of Biomaterials \& Engineering (WIBEZD2017001-03), Visiting Scholar Foundation of Key Laboratory of Biorheological Science and Technology (Chongqing University), Ministry of Education(No. CQKLBST-2018-005).

\section{Reference}

1. V. Sanna, N. Pala and M. Sechi, Int. J. Nanomed., 2014, 9, 467.

2. X. Q. Zhang, X. Xu, N. Bertrand, E. Pridgen, A. Swami and O. C. Farokhzad, Adv. Drug Deliver. Rev., 2012, 64, 1363-1384.

3. S. M. Janib, A. S. Moses and J. A. MacKay, Adv. Drug Deliver. Rev., 2010, 62,1052-1063.

4. D. Yoo, J H. Lee, T. H. Shin and J. Cheon, Accounts Chem. Res., 2011, 44, 863-874.

5. D. E. Lee, H. Koo, I. C. Sun, J. H. Ryu, K. Kim and I. C. Kwon, Chem. Soc. Rev., 2012, 41, 2656-2672.

6. M. Ferrari, Nat. Rev. Cancer, 2005, 5,161.

7. Y. Matsumura and H. Maeda, Cancer Res., 1986, 46, 6387-6392.

8. A. Taylor, K. M. Wilson, P. Murray, D. G. Fernig and R. Lévy, Chem. Soc. Rev., 2012, 41, 2707-2717.
9. D. Niu, Y. Li and J. Shi, Chem. Soc. Rev., 2017, 46, 569-585.

10. G. Bozzuto and A. Molinari, Inter. J. Nanomed., 2015, 10, 975.

11. K. H. Bae, H. J. Chung and T. G. Park, 2011, 31, 295-302.

12. M. J. D. Clift and V. Stone, Theranostics, 2012, 2, 668.

13. S. Y. Wu, G. Lopez-Berestein, G. A. Calin and A. K. Sood, Sci. Transl. Med., 2014, 6, 240ps247-240ps247.

14. K. A. Whitehead, R. Langer and D. G. Anderson, Nat. Rev. Drug Discov., 2009, 8, 129.

15. D. W. Pack, A. S. Hoffman and S. Pun, P. S. Nat. Rev. Drug Discov, 2005, 4, 581.

16. J. Shen, W. Zhang, R. Qi, Z. W. Mao and H. Shen, Chem. Soc. Rev,, 2018, 47, 1969-1995.

17. S. T. Kim, A. Chompoosor, Y. C. Yeh, S. S. Agasti, D. J. Solfiell and V. M. Rotello, Small, 2012, 8, 3253-3256.

18. J. Shen, H. C. Kim, C. Mu, E. Gentile, J. Mai and J. Wolfram et al. , Advanced Healthc. Mater., 2014, 3, 1629-1637.

19. I. d'Angelo, G. Costabile, E. Durantie, P. Brocca, V. Rondelli and A. Russo et al. , J. Aerosol Med. Pulm. D., 2018, 31, 170-181.

20. J. Wolfram, H. Shen and M. Ferrari, J. Control. Release, 2015, 219, 406-415.

21. J. Shen, R. Xu, J. Mai, H. C. Kim, X. Guo and G. Qin et al. , ACS Nano, 2013, 7, 9867-9880.

22. J. Shen, H.-C. Kim, H. Su, F. Wang, J. Wolfram and D. Kirui et al. , Theranostics, 2014, 4, 487.

23. S. Hoelder, P. A. Clarke and P. Workman, Mol. Oncol., 2012, 6: 155-176.

24. M. M. Gottesman, T. Fojo and S. E. Bates, Nat. Rev. Cancer, 2002, 2, 48.

25. M. Overchuk and G. Zheng, Biomaterials, 2018, 156, 217-237.

26. J. E. Lee, N. Lee, T. Kim, J. Kim and T. Hyeon, Accounts Chem Res, 2011, 44,893-902.

27. R. Xu, G. Zhang, J. Mai, X. Deng, V. Segura-Ibarra and S. Wu, Nat. Biotechnol., 2016, 34, 414.

28. Z. Liu and X. J. Liang, Theranostics, 2012, 2, 235.

29. X. Deng, P. Qin, M. Luo, E. Shao, H. Zhao and X. Yang, Langmuir, 2013, 29, 6815-6822.

30. M. Ferrari, Nat. Rev. Cancer, 2005, 5, 161

31. J. Shen, H. Liu, C. Mu, J. Wolfram, W. Zhang and H. C. Kim, Nanoscale, 2017, 9, 5329-5341.

32. J. Shen, J. Wolfram, M. Ferrari and H. Shen, Materials today (Kidlington, England), 2017, 20, 95-97.

33. J. R. Vargas, E. G. Stanzl, N. N. H. Teng and P. A. Wender, Mol Pharmaceut., 2014, 11, 2553-2565.

34. A. K Iyer, J. He and M. M Amiji, Curr. Med. Chem., 2012, 19, 3230-3240.

35. T. Lammers, S. Aime, W. E. Hennink, G. Storm and F. Kiessling, Accounts. Chem.. Res., 2011, 44, 1029-1038.

36. W. C. W. Chan and S. Nie, Science, 1998, 281, 2016-2018.

37. P. Zrazhevskiy, M. Sena and X. Gao, Chem. Soc. Rev., 2010, 39, 4326-4354.

38. V. Biju, S. Mundayoor, R. V. Omkumar, A. Anas and M. Ishikawa Biotechnol. Adv., 2010, 28, 199-213.

39. A. K. Patri, J. F. Kukowska-Latallo and J. R. Baker Jr, Adv. Drug Deliver. Rev., 2005, 57, 2203-2214.

40. M. X. Zhao, Q. Xia, X. D. Feng, X. H. Zhu, Z. W. Mao, L. N. Ji et al. Biomaterials, 2010, 31, 4401-4408.

41. J. M. Li, M. X. Zhao, H. Su, Y. Y. Wang, C. P. Tan and L. N. Ji et al. , Biomaterials, 2011, 32, 7978-7987.

42. M. X. Zhao, H. Su, Z. W. Mao and L. N. Ji, J. Lumin., 2012, 132, 16-22.

43. M. S. Muthu, D. T. Leong, L. Mei and S. S. Feng, Theranostics, 2014, 4, 660.

44. X. Ma, Y. Zhao and X. J. Liang, Accounts. Chem.. Res., 2011, 44,1114-1122.

45. J. Zhao, Y. Mi and S. S. Feng, Nanomedicine, 2013, 8, 859-862.

46. J. M. Li, Y. Y. Wang, M. X. Zhao, C. P. Tan, Y. Q. Li and X. Y. Le, Biomaterials, 2012, 33, 2780.

47. J. Shen, H.-C. Kim, J. Wolfram, C. Mu, W. Zhang and H. Liu, Nano Lett., 2017, 17, 2913-2920.

Publisher's Note Engineered Science Publisher remains neutral with regard to jurisdictional claims in published maps and institutional affiliations. 